\title{
Well placement optimization for offshore oilfield based on Theil index and differential evolution algorithm
}

\author{
Hongwei Chen ${ }^{1} \cdot$ Qihong Feng $^{1} \cdot$ Xianmin Zhang $^{1} \cdot$ Sen Wang ${ }^{1} \cdot$ Wensheng Zhou ${ }^{2} \cdot$ \\ Chen Liu ${ }^{2}$
}

Received: 2 September 2017 / Accepted: 20 November 2017/Published online: 25 November 2017

(c) The Author(s) 2017. This article is an open access publication

\begin{abstract}
Proper well placement should match the heterogeneity of reservoir and development schedule, in which case the displacement is balanced. A balanced displacement process can achieve a good development performance of reservoir. Present parameters to evaluate the displacement balanced degree are not fit for high water-cut oilfields and need a specific reservoir simulator. In this work, a well placement optimization method for offshore oilfield is established. A displacement balanced degree evaluation method is proposed based on Theil index. By using minimum Theil index as the objective function, well placement optimization model for offshore oilfield is built. In this model, constraints about the available infilling scope and minimum inter-well distance are considered to find the realizable optimal location. This optimization model is solved by a derivative-free optimization algorithm, differential evolution algorithm. This method is applied and validated in a semisynthetic offshore oilfield to find optimal well locations. Results demonstrate that this proposed method can find the optimal well placement for offshore oilfield to achieve a more balanced displacement and a higher oil recovery.
\end{abstract}

Keywords Well placement - Optimization model . Offshore oilfield · Theil index · Differential evolution algorithm

Qihong Feng

fengqihong.upc@gmail.com

1 School of Petroleum Engineering, China University of Petroleum (East China), Qingdao 266580, Shandong, China

2 CNOOC Research Institute, Beijing 100027, China

\section{List of symbols}

$c_{\mathrm{r}} \quad$ Rock compressibility

CR Crossover probability

D Problem dimension

Dis Inter-well distance

$f \quad$ Objective function in DE algorithm

$F \quad$ Mutation factor

$g_{k} \quad$ Total number of injector-producer lines in the $k$ th injection-production group

$H \quad$ Vertical distance between the wellhead tower and the top surface of reservoir

$i \quad i$ th injector-producer line

$j \quad j$ th optimized well

$k \quad k$ th injection-production group

$K$ Total number of injection-production groups

$L_{i} \quad$ Length of $i$ th injector-producer line

max Maximum

min Minimum

$N \quad$ Total number of injector-producer lines

$N_{k} \quad$ Number of injector-producer lines in the $k$ th injection-production group

NP Population size in DE algorithm

$p \quad p$ th vector $\mathbf{x}$ in DE algorithm

$q \quad$ Component number

$q_{\text {rand }}$ A random integer

$t \quad$ Iteration step

$T \quad$ Theil index

$T_{\mathrm{b}} \quad$ Displacement balanced degree between injectionproduction groups

$T_{\mathrm{w}} \quad$ Displacement inequality degree within injectionproduction groups

$U \quad$ Uniformly distributed random number sample

v Mutation vector

$\mathbf{x} \quad$ Target vector

$x_{i} \quad$ Oil saturation at $i$ th injector-producer line 
$x_{\mathrm{h}} \quad$ Coordinates of heel position

$x_{t} \quad$ Coordinates of toe position

$\mathbf{x}_{r 1}^{t} \quad$ Chosen vector at iteration $t$

$\mathbf{x}_{r 2}^{t} \quad$ Chosen vector at iteration $t$

$\mathbf{x}_{r 3}^{t} \quad$ Chosen vector at iteration $t$

$X_{i} \quad$ The equivalent oil saturation of ith injectorproducer line

$X_{k} \quad$ Total oil saturation of the $k$ th injection-production group

$\bar{X} \quad$ Average equivalent oil saturation of all injectorproducer lines

$y_{\mathrm{h}} \quad$ Coordinates of heel position

$y_{\mathrm{t}} \quad$ Coordinates of toe position

y Trial vector

$Y_{k} \quad$ Total oil saturation in the reservoir

$z_{\mathrm{h}} \quad$ Coordinates of heel position

$z_{t} \quad$ Coordinates of toe position

$\mu_{\mathrm{o}} \quad$ Oil viscosity

$\mu_{w} \quad$ Water viscosity

$\rho_{\mathrm{o}} \quad$ Oil density

$\rho_{\mathrm{w}} \quad$ Water density

$\phi \quad$ Porosity

\section{Introduction}

Determining the optimal well placement plays an important role in the oilfield development (Humphries and Haynes 2015; Arinkoola et al. 2015; Dossary and Nasrabadi 2016; Chen et al. 2017a; Ogbeiwi et al. 2017). A proper well placement can yield a balanced displacement and large financial return (Siavashi et al. 2016; Awotunde and Naranjo 2014; Ogbeiwi et al. 2017). And a balanced displacement process can achieve a good development performance of reservoir (Zhou et al. 2017). However, due to the heterogeneity of reservoir and fluid property, the displacement is often unbalanced. Many parameters are used to measure the displacement balanced degree. Li et al. (2006) determined the well placement according to the water breakthrough time in each injection-production line. They adjusted the well placement to make well placement match with the geological vector. Zhou et al. (2017) proposed a well placement optimization method to realize maximum equilibrium displacement. In their method, displacement balanced degree was measured by the variance of the water breakthrough time. Siavashi et al. (2016) established a well placement optimization method to achieve the distribution of streamline be more uniform. The objective function was constructed by the coefficient of variance of streamline number and time of flight to measure the displacement balanced degree. This method made sure that most regions of the reservoir could be swept. Jesmani et al. (2016) analyzed the optimal well placement according to the time of flight. The optimal well locations in their research could obtain an even time of flight in each injection-production line. However, most waterflooding oilfields have entered into high water-cut stage and many producers have seen the water. The water breakthrough time is not fit for high water-cut oilfields. Besides, time of flight can be obtained by the specific reservoir simulator which would limit its application to a certain degree. The average oil saturation of injectionproduction group can reflect the displacement degree (Feng et al. 2016; Chen et al. 2017a). It can be used in any development stage of oilfield. And most reservoir simulator can obtain the oil saturation during simulating process. Therefore, in this work oil saturation is used to reflect the displacement degree of each injection-production region. The distribution of remaining oil in the reservoir can reflect the displacement balanced degree. For the whole reservoir, the displacement balanced degree depends on the displacement balanced degree between injection-production groups and within injection-production groups. However, the evaluation methods in current researches cannot provide the comprehensive evaluation. Theil index is widely used to describe the inequality characteristic (Alcantara and Duro 2004; Padilla and Serrano 2006; Clarke-Sather et al. 2011). It describes the total amount of inequality in a distribution by the extent of inequality within groups and between them (Padilla and Serrano 2006). Given the same evaluation range, Theil index can effectively evaluate the displacement balanced degree of the oilfield. To the best of our knowledge, Theil index has not been applied in the evaluation of displacement balanced degree.

Well placement optimization problem is usually complex, nonconvex and multimodal (Awotunde and Naranjo 2014; Wang et al. 2016). In order to solve well placement optimization problem, both gradient-based and derivativefree optimization algorithms are applied (Yeten et al. 2002; Bangerth et al. 2006; Taware et al. 2012; Bouzarkouna et al. 2012; Nwankwor et al. 2013; Awotunde 2014; Isebor et al. 2014; Humphries and Haynes 2015; Carosio et al. 2015; Khademi and Karimaghaee 2016; Bagheri and Masihi 2016), such as finite difference gradient (FDC), simultaneous perturbation stochastic approximation (SPSA), genetic algorithm (GA), particle swarm optimization (PSO) algorithm, covariance matrix adaptation evolution strategy (CMA-ES) algorithm and differential evolution (DE) algorithm. Due to the simple metaheuristic mechanism, DE algorithm has shown good performance in solving the real parameter optimization problem and global optimization problem (Carosio et al. 2015). It has been used to deal with well placement optimization problem in many researches (Awotunde 2014; Carosio et al. 2015). In 
this paper, we apply DE algorithm to solve the well placement optimization problem in offshore oilfield.

This paper establishes a well placement optimization method for offshore oilfield based on Theil index and differential evolution algorithm. Firstly, the evaluation method of displacement balanced degree is proposed based on Theil index, which can provide a detailed evaluation of displacement balanced degree in the whole reservoir, within injector-producer well groups and between them. Then, well placement optimization model for offshore oilfield is established to realize the maximum equilibrium displacement, which uses minimum Theil index as the objective function and is solved by DE algorithm. Finally, we apply this well placement optimization method to find optimal locations of infilling wells in a semisynthetic offshore oilfield. This paper can provide an well placement optimization method for offshore oilfield to realize the balanced displacement.

\section{Methodology}

In order to find the optimal well placement for offshore oilfield to realize the balanced displacement, three basic elements are needed: (1) displacement balanced degree evaluation method; (2) well placement optimization model; and (3) optimization algorithm. In this section, we apply Theil index to evaluate the displacement balanced degree. Then, given the characteristics of offshore oilfield, we establish the well placement optimization model. To deal with this discontinuous and multilocal optimums optimization problem, a derivative-free optimization, differential evolution (DE) algorithm, is used to solve the problem.

\section{Displacement balanced degree evaluation based on Theil index}

Theil index is an efficient inequality evaluation index. It can provide a detailed description of inequality within groups and between them (Alcantara and Duro 2004; Padilla and Serrano 2006; Clarke-Sather et al. 2011). The displacement unbalanced degree of the whole reservoir can be decomposed into two synthetic components, i.e., within injection-production groups component and between injection-production groups component. Based on Theil index, the displacement balanced degree evaluation method is built.

The displacement balanced degree of the whole reservoir can be defined as follows:
$T=\frac{1}{N} \sum_{i=1}^{N} \frac{X_{i}}{\bar{X}} \ln \frac{X_{i}}{\bar{X}}$

where $T$ is the Theil index denoting the displacement balanced degree of the whole reservoir; $N$ is the total number of injector-producer lines; $X_{i}$ is the equivalent oil saturation of $i$ th injector-producer line; and $\bar{X}$ denotes average equivalent oil saturation of all injector-producer lines. Because water flooding performance is mainly influenced by the parameters on mainstream line (Zhou et al. 2017; Chen et al. 2017b), we use oil saturation in injector-producer line to reflect the displacement process. Because oil saturation is not a constant along the injectorproducer line, the equivalent oil saturation of each injector-producer line $X_{i}$ is calculated by:

$X_{i}=\frac{1}{L_{i}} \int_{l} x_{i}(x, y) \mathrm{d} x_{i}$

where $L_{i}$ is the length of $i$ th injector-producer line; $x_{i}$ ($x, y)$ is the oil saturation at $(x, y)$ of $i$ th injector-producer line.

The displacement balanced degree of the whole water flooding reservoir can be decomposed into between injection-production groups and within injection-production groups as follows:

$T=T_{\mathrm{b}}+T_{\mathrm{w}}$

where $T_{\mathrm{b}}$ represents the displacement balanced degree between injection-production groups; $T_{\mathrm{w}}$ denotes the displacement inequality degree within injection-production groups.

$T_{\mathrm{b}}$ is calculated as:

$T_{\mathrm{b}}=\sum_{k=1}^{K} \frac{X_{k}}{Y_{k}} \ln \frac{X_{k} / Y_{k}}{N_{k} / N}$

where $K$ is the total number of injection-production group; $X_{k}$ is the total oil saturation of the $k$ th injection-production group; $Y_{k}$ is the total oil saturation in the reservoir; and $N_{k}$ is the number of injector-producer lines in the $k$ th injection-production group.

$T_{\mathrm{w}}$ is calculated as:

$T_{\mathrm{w}}=\sum_{k=1}^{K} \frac{X_{k}}{Y_{k}}\left(\sum_{i \in g_{k}}^{N_{k}} \frac{X_{i}}{X_{k}} \ln \frac{X_{i} / X_{k}}{1 / N_{k}}\right)$

where $g_{k}$ is the total number of injector-producer lines in the $k$ th injection-production group.

Therefore, parameters $T, T_{\mathrm{b}}$ and $T_{\mathrm{w}}$ can provide a detailed description of displacement balanced degree in the whole reservoir, within injector-producer well groups and between them. 


\section{Optimization model based on Theil index}

The range of Theil index is from zero to one. When the displacement of the reservoir is absolutely balanced, in which case all injector-producer line's oil saturations are equal, Theil index attains its minimum (zero). One represents that the displacement is extremely unbalanced. The objective of well placement optimization is to realize maximum equilibrium displacement (Zhou et al. 2017). Therefore, the objective function of well placement optimization model is the minimum difference of injectorproducer lines' oil saturations which can be formulated as:

$\min T=\frac{1}{N} \sum_{i=1}^{N} \frac{X_{i}}{\bar{X}} \ln \frac{X_{i}}{\bar{X}}$

The optimization variables are the well placement. Wells are drilled from wellhead tower in offshore oilfield (Marir et al. 2015), and they are usually in a cluster type (Cao et al. 2016). For this kind of well, the available infilling scope is determined by the wellhead tower. If we do not take this constraint into account, the optimized well may be unrealized in offshore oilfield development. Besides, many wells are drilled from a same wellhead tower in offshore oilfield for cluster well group. The interwell distance should not be too small in which case wellbore anti-collision could occur. Therefore, constraints about available infilling scope and minimum inter-well distance should be considered in well placement optimization for offshore oilfield.

Six parameters $\left(x_{\mathrm{h}}, y_{\mathrm{h}}, z_{\mathrm{h}}, x_{\mathrm{t}}, y_{\mathrm{t}}\right.$ and $\left.z_{\mathrm{t}}\right)$ are usually used to describe the well location in three-dimensional reservoir. $x_{\mathrm{h}}, y_{\mathrm{h}}$ and $z_{\mathrm{h}}$ are the coordinates of heel position, and $x_{\mathrm{t}}, y_{\mathrm{t}}$ and $z_{\mathrm{t}}$ are the coordinates of toe position. The constraint of available infilling scope can be given as:

$$
\left\{\begin{array}{c}
x_{\min } \leq x_{\mathrm{h}} \leq x_{\max } \\
y_{\min } \leq y_{\mathrm{h}} \leq y_{\max } \\
z_{\min } \leq z_{\mathrm{h}} \leq z_{\max } \\
x_{\min } \leq x_{\mathrm{t}} \leq x_{\max } \\
y_{\min } \leq y_{\mathrm{t}} \leq y_{\max } \\
z_{\min } \leq z_{\mathrm{t}} \leq z_{\max }
\end{array}\right.
$$

where $x_{\min }, y_{\min }, z_{\min }$ and $x_{\max }, y_{\max }, z_{\max }$ are the minimum and maximum boundary of available infilling scope.

Minimum inter-well distance constraint has been studied in many researches (Awotunde and Naranjo 2014; Jesmani et al. 2016; Zhang et al. 2017). According to the minimum distance between two line segments, the minimum interwell distance Dis well $_{\text {can }}$ be obtained. Therefore, the constraint of minimum inter-well distance can be formulated as:

Dis $_{\text {well }} \geq$ Dis $_{\text {min }}$ where $\mathrm{Dis}_{\min }$ is the minimum inter-well distance.

Therefore, the well placement optimization problem for offshore oilfield can be stated as: determining the optimal well placement to realize the maximum equilibrium displacement, i.e., minimize the difference of injector-producer lines' oil saturations denoted by Theil index objective function, subjected to available infilling scope and minimum inter-well distance. It can be formulated as:

$\min T=\frac{1}{N} \sum_{i=1}^{N} \frac{X_{i}}{\bar{X}} \ln \frac{X_{i}}{\bar{X}}$

subject to

$\left\{\begin{array}{c}x_{\min } \leq x_{\mathrm{h}} \leq x_{\max } \\ y_{\min } \leq y_{\mathrm{h}} \leq y_{\max } \\ z_{\min } \leq z_{\mathrm{h}} \leq z_{\max } \\ x_{\min } \leq x_{\mathrm{t}} \leq x_{\max } \\ y_{\min } \leq y_{\mathrm{t}} \leq y_{\max } \\ z_{\min } \leq z_{\mathrm{t}} \leq z_{\max } \\ \text { Dis }_{\mathrm{well} j} \geq \text { Dis }_{\min }\end{array}\right.$

For the offshore oilfield which the infilling well pattern is known, the well placement optimization can be simplified to only having the constraint of infilling well scope.

\section{Optimization algorithm}

In this research, DE algorithm is applied to solve the well placement optimization problem. DE algorithm outperforms many other optimization algorithms because of its excellent performance in convergence speed and robustness (Huang et al. 2006). Several papers have applied DE to solve optimization problems including well placement optimization and well control optimization (Carosio et al. 2015). Therefore, we use DE algorithm to optimize the well placement.

DE algorithm is an evolutionary algorithm with the iterative process of mutation, crossover and selection operators (Carosio et al. 2015). A population of $D$-dimensional vectors inside the problem bounds is generated as the initial population firstly. The objection functions for all the vectors are evaluated, and some candidate solutions are maintained at each generation. Besides, some candidates are chosen to create new candidates. The optimization process stops until a stopping condition is satisfied.

In DE evaluation strategy, the each vector $\mathbf{x}_{p}$ in a population $N P$ is used to generate mutation vector $\mathbf{v}_{p}$ by mutation operator. Three vectors are randomly selected for the population to generate the mutation vector $\mathbf{v}_{p}$ at iteration $t$ which is calculated by (Nwankwor et al. 2013). 
$\mathbf{v}_{p}^{t+1}=\mathbf{x}_{r 1}^{t}+F\left(\mathbf{x}_{r 1}^{t}-\mathbf{x}_{r 3}^{t}\right)$

where $\mathbf{x}_{r 1}^{t}, \mathbf{x}_{r 2}^{t}$ and $\mathbf{x}_{r 3}^{t}$ are three chosen vectors at iteration $t$; $F$ is the mutation factor with the range of $0-2$. This process is called mutation.

Then, the trial vector $\mathbf{y}_{p, q}^{t+1}$ is calculated according to the target vector $\mathbf{x}_{p, q}^{t}$ and the mutation vector $\mathbf{x}_{p . q}^{t+1}$ by crossover operator (Carosio et al. 2015):

$\mathbf{y}_{p, q}^{t+1}= \begin{cases}\mathbf{v}_{p, q}^{t+1}, & \text { if } U(0,1) \leq C R \text { or } q=q_{\mathrm{rand}} \\ \mathbf{x}_{p, q}^{t}, & \text { if } U(0,1)>C R \text { or } q \neq q_{\mathrm{rand}}\end{cases}$

where $q \in\{1,2, \ldots, \mathrm{NP}\} ; U(0,1)$ is a uniformly distributed random number sample between 0 and $1 ; C R$ is the crossover probability between 0 and $1 ; q_{\text {rand }}$ is a random integer from 0 to NP.

The vectors for the next iteration $t+1$ are generated by comparing the target vector $\mathbf{x}_{p, q}^{t}$ with the trial vector $\mathbf{y}_{p . q}^{t+1}$. If the objective value of trial vector $\mathbf{y}_{p . q}^{t+1}$ is bigger than objective value of trial vector $\mathbf{x}_{p, q}^{t}$, trial vector $\mathbf{y}_{p . q}^{t+1}$ will replace the trial vector $\mathbf{x}_{p, q}^{t}$. Otherwise, the $\mathbf{x}_{p, q}^{t}$ will be maintained in the next iteration. This process is conducted by selection operator:

$\mathbf{x}_{p, q}^{t+1}=\left\{\begin{array}{cc}\mathbf{y}_{p, q}^{t+1}, & \text { if } f\left(\mathbf{y}_{p, q}^{t+1}\right) \leq f\left(\mathbf{x}_{p, q}^{t}\right) \\ \mathbf{x}_{p, q}^{t}, & \text { if } f\left(\mathbf{y}_{p, q}^{t+1}\right)>f\left(\mathbf{x}_{p, q}^{t}\right)\end{array}\right.$

where $f$ is the objective function; $\mathbf{x}_{p . q}^{t+1}$ is the vector for iteration $t+1$.

During the well placement optimization process, a reservoir numerical simulator ECLIPSE 100 (GeoQuest 2010) is used to calculate the remaining oil distribution under different well placements. ECLIPSE 100 is a fullimplicit black oil simulator. It can calculate the remaining oil and development indexes by inputting the reservoir parameters and well placements. We use MATLAB software (MATLAB 2013) to perform the DE algorithm optimization process and apply ECLIPSE software to calculate the objective function. The flow diagram for well placement optimization based on Theil index and DE algorithm in offshore oilfield can be seen in Fig. 1.

\section{Results and discussion}

In this work, the well placement for offshore oilfield is optimized based on Theil index and DE algorithm to realize maximum equilibrium displacement. A semisynthetic offshore oilfield model is applied in this section.

It is a three-dimensional two-phase reservoir model. There are a total of $140 \times 140 \times 3$ uniform grid blocks,

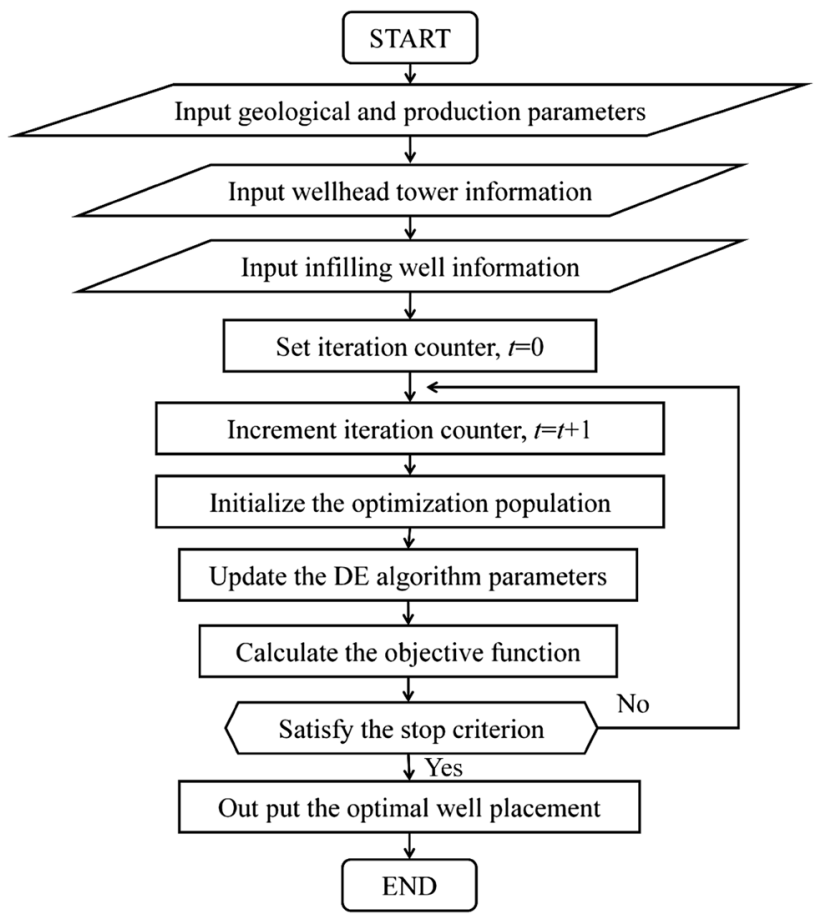

Fig. 1 Flowchart of well placement optimization based on Theil index and DE algorithm

and the reservoir is $1400 \times 1400 \times 30 \mathrm{~m}^{3}$. The permeability distribution of each layer is shown in Fig. 2a. There are 4 regular inverted nine-spot patterns with 16 producers and 9 injectors. The position of each well can be seen in Fig. 2. They are controlled by constant liquid production rate and constant injection rate, respectively. We assume this reservoir has been developed for a certain time, and the present oil saturation distribution is shown in Fig. $2 b$. Other data of the reservoir model are listed in Table 1. The oil and water relative permeability curves are plotted in Fig. 3. And the capacity pressure is ignored.

As shown in Fig. 3b, remaining oil is mainly distributed between the producers. In order to extract more oil and improve the development performance, infilling wells are needed to be drilled. For the offshore oilfield with inverted nine-spot pattern, the successful infilling pattern is to convert the producer which locates between injectors to injector and the infilling producers are drilled between producers. This sketch of infilling pattern can be seen in Fig. 4. Therefore, 8 infilling producers are drilled in this case. We suppose all wells in this case are vertical wells. These infilling producers are controlled by a constant liquid production rate of $160 \mathrm{~m}^{3} /$ day. The reservoir produces 7200 days from now on.

The locations of infilling wells are determined by two methods. One is the regular infilling method. For this 
Fig. 2 Permeability and present oil saturation distribution
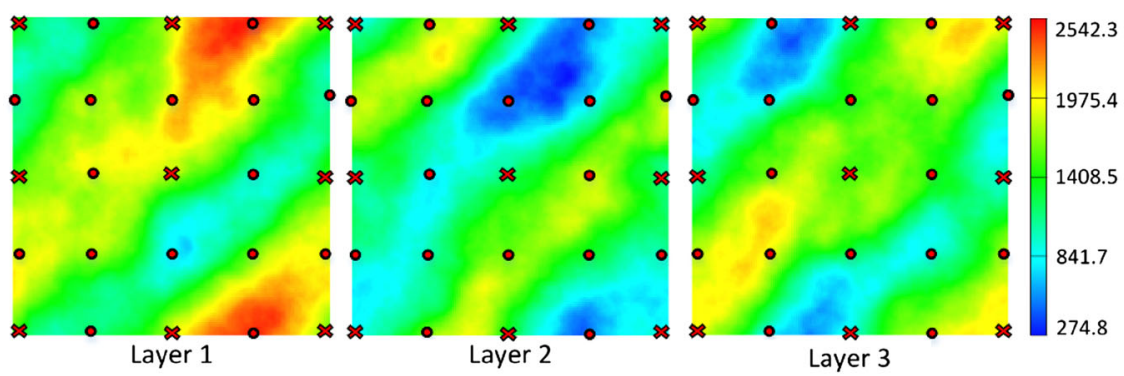

(a) Permeability distribution
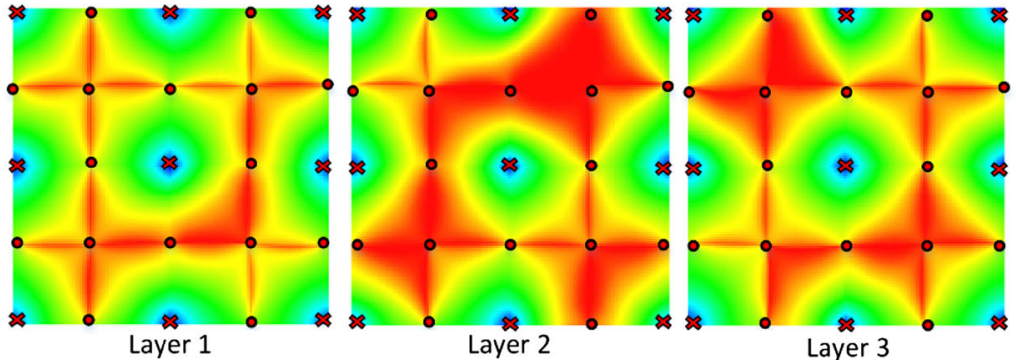

0.700

0.575

0.450

0.325 * Injector

Layer 1

(b) Present oil saturation distribution
0.200 - Producer $2.3\left(10^{-3} \mu \mathrm{m}^{2}\right)$

75.4

8.5

1.7

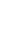

(a) Permeability distribution
Table 1 Reservoir conditions and rock and fluid properties

\begin{tabular}{lll}
\hline Parameter & Symbol & Value \\
\hline Porosity & $\phi$ & 0.3 \\
Oil viscosity & $\mu_{\mathrm{o}}$ & $0.005 \mathrm{~Pa} \cdot \mathrm{s}$ \\
Water viscosity & $\mu_{\mathrm{w}}$ & $0.001 \mathrm{~Pa} \cdot \mathrm{s}$ \\
Oil density & $\rho_{\mathrm{o}}$ & $0.947 \mathrm{~g} / \mathrm{m}^{3}$ \\
Water density & $\rho_{\mathrm{w}}$ & $0.998 \mathrm{~g} / \mathrm{m}^{3}$ \\
Rock compressibility & $c_{\mathrm{r}}$ & $5 \times 10^{-4} \mathrm{MPa}^{-1}$ \\
\hline
\end{tabular}

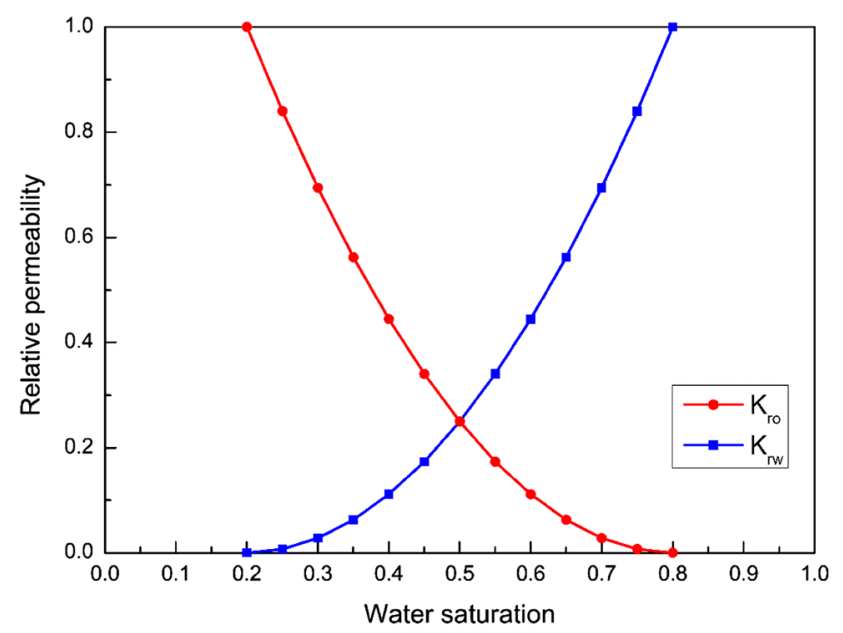

Fig. 3 Oil and water relative permeability curve

method, infilling wells locate in the middle of the producers. The other method is the optimization method. Locations of these infilling wells are optimized by the

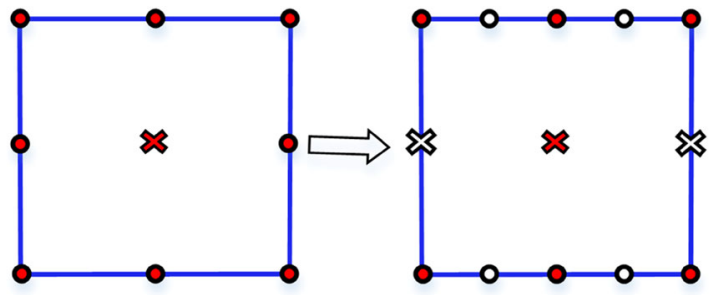

Initial well pattern Infilling well pattern

$\approx$ Injector $\bullet$ Producer $\odot$ Infilling well $\approx$ Convert producer to injector

Fig. 4 Sketch of infilling pattern in inverted nine-spot pattern

proposed optimization method in this work. The coordinates of wellhead tower in $x-y$ plane are $(70,70)$, and the vertical distance between the wellhead tower and the top surface of reservoir is $1000 \mathrm{~m}$. The maximum angle of the line between the wellhead tower and the maximum infilling scope making with the vertical plane is $\pi / 4$. And the minimum inter-well distance is $80 \mathrm{~m}$. According to the infilling pattern of this case, locations of infilling wells can be optimized only considering the infilling scope constraint. For this method, the parameters of DE algorithm are listed in Table 2. The problem dimension $(D)$ is 16 . The number of population (NP) we set is 50 . The mutation factor $(F)$ is equal to 0.5 , and the crossover probability (CR) is 0.9 . The total number of iterations is 30 . In order to overcome the stochastic property of DE algorithm, 10 runs are performed. Figure 5 shows the convergence progress of the proposed optimization method over 10 runs.

Figures 6 and 7, respectively, show the locations of infilling wells and oil saturation distribution after 
Table 2 DE algorithm parameters

\begin{tabular}{lllll}
\hline$D$ & NP & $F$ & CR & Iterations \\
\hline 16 & 50 & 0.5 & 0.9 & 30 \\
\hline
\end{tabular}

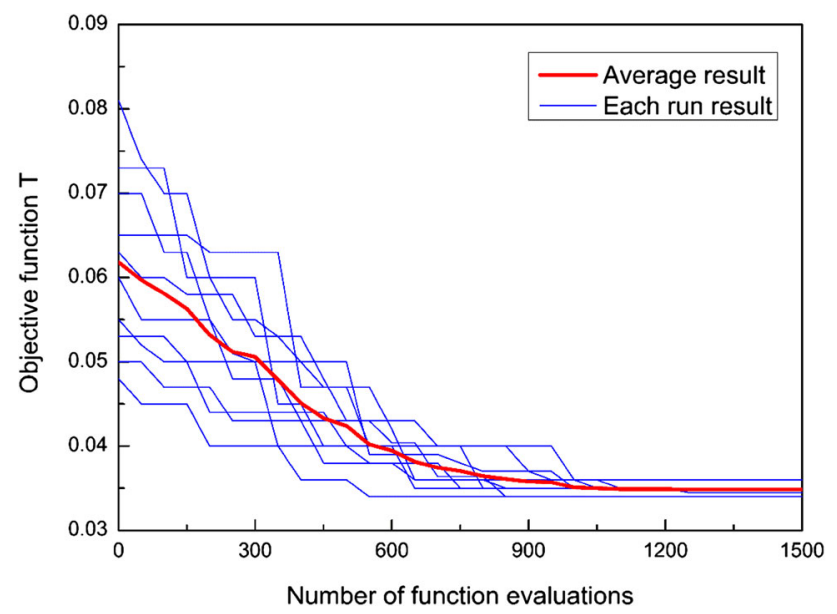

Fig. 5 Convergence curve for all ten runs versus number of simulations

7200 days for two infilling methods. The displacement balanced degrees under the regular infilling method and the proposed optimization method are listed in Table 3. From
Table 3, it can be observed that the displacement balanced degree of the whole reservoir and between injection-production groups is decreased by 14.29 and $20.00 \%$ after optimization, respectively. In comparison with the regular infilling method, the oil saturation distribution under the proposed optimization method is more balanced as shown in Figs. 6 and 7.

The well production performance comparison of the regular infilling method and the proposed optimization method is shown in Fig. 8. It illustrates that the cumulative oil production under the proposed optimization method can increase $4.3 \%$ cumulative oil production than the cumulative oil production under the regular infilling method. It validates that the proposed optimization method can effectively improve the offshore oilfield development performance.

Therefore, well placement optimization established in this work can find the optimal well placement to increase oil production. It can realize the displacement in water flooding reservoir be more balanced which improves the production performance.

\section{Conclusions}

1. A well placement optimization method for offshore oilfield has been established based on Theil index and
Fig. 6 Oil saturation distribution under regular infilling method

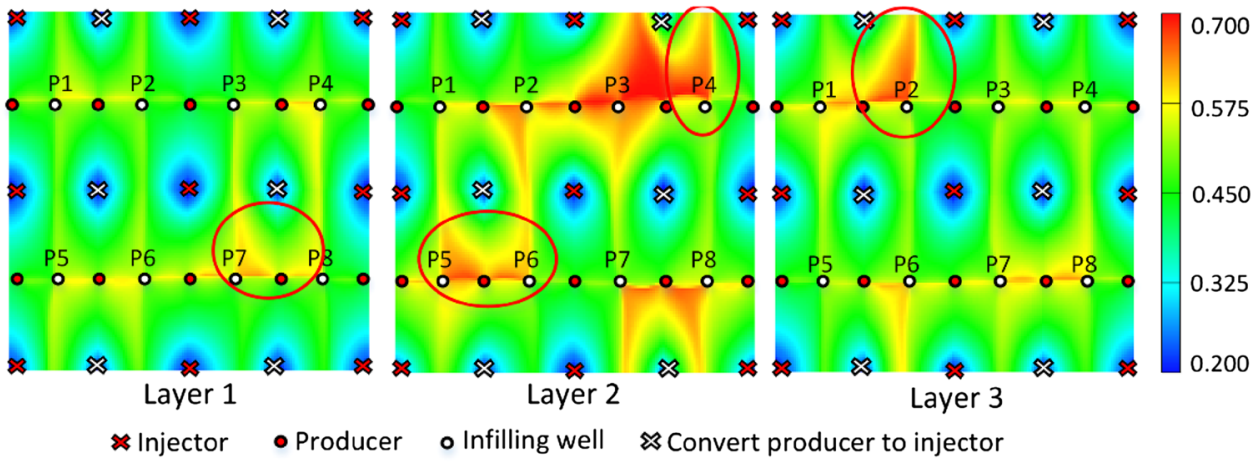

Fig. 7 Oil saturation distribution under proposed optimization method

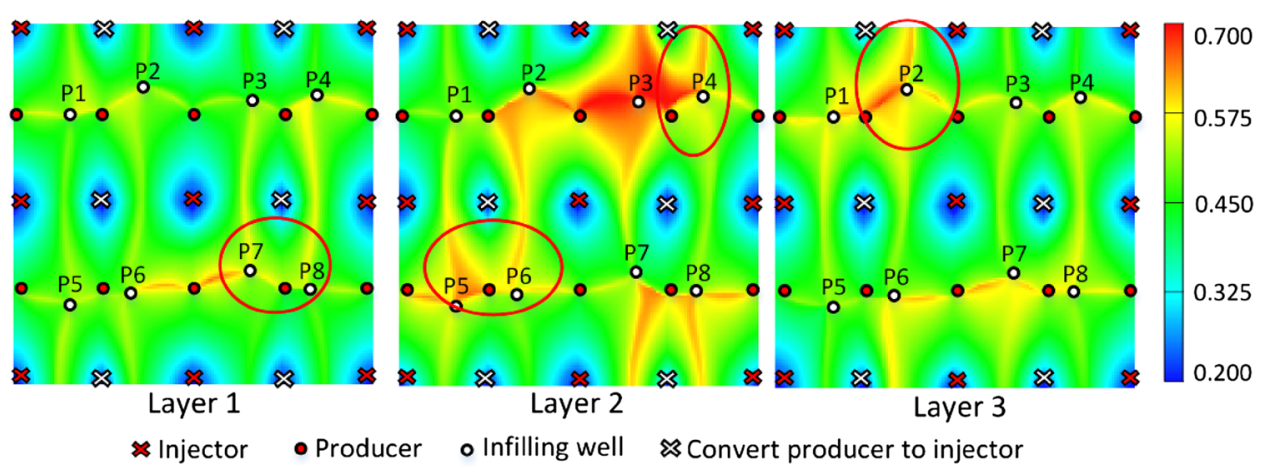

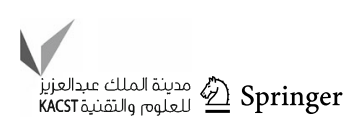


Table 3 Displacement balanced degree of different infilling method after 7200 days

\begin{tabular}{llll}
\hline Infilling method & $T$ & $T_{\mathrm{b}}$ & $T_{\mathrm{w}}$ \\
\hline Regular infilling method & 0.0040 & 0.0030 & 0.0010 \\
Proposed optimization method & 0.0035 & 0.0024 & 0.0012 \\
\hline
\end{tabular}

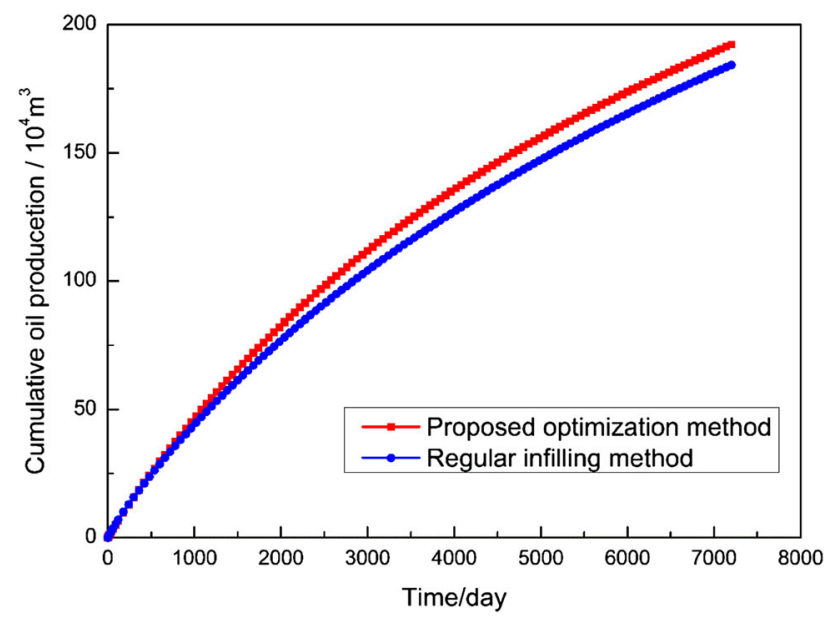

Fig. 8 Cumulative oil production comparison curve of different infilling method

differential evolution algorithm. The target of this method is to realize the maximum equilibrium displacement of the offshore oilfield.

2. A well placement optimization model for offshore oilfield is built. Minimum Theil index is used as the objective function to reflect the displacement balanced degree. Considering the characteristics of offshore oilfield, available infilling scope and minimum interwell distance have been taken into account during well placement optimization procedure. A derivative-free optimization algorithm, DE algorithm, is applied to solve this well placement optimization problem by being combined with a reservoir numerical simulator.

3. The locations of infilling wells in a semisynthetic offshore oilfield model are optimized by the established method. Results demonstrate that this method can find the optimal well placements to increase oil production. The displacement under the optimal well placements can be more balanced which can improve the production performance.

Acknowledgements This research is supported by the National Science and Technology Major Project of China (Grant No. 2016ZX05025001-006), Program for Changjiang Scholars and Innovative Research Team in University (IRT1294), National Natural Science Foundation of China (51474233), National Postdoctoral Program for Innovative Talents (BX201600153), China Postdoctoral
Science Foundation (2016M600571) and Qingdao Postdoctoral Applied Research Project (2016218).

Open Access This article is distributed under the terms of the Creative Commons Attribution 4.0 International License (http:// creativecommons.org/licenses/by/4.0/), which permits unrestricted use, distribution, and reproduction in any medium, provided you give appropriate credit to the original author(s) and the source, provide a link to the Creative Commons license, and indicate if changes were made.

\section{References}

Alcantara V, Duro J (2004) Inequality of energy intensities across OECD countries: a note. Energy Policy 32(11):1257-1260

Arinkoola A, Onuh H, Ogbe D (2015) Quantifying uncertainty in infill well placement using numerical simulation and experimental design: case study. J Pet Explor Prod Technol 8(2):1-15

Awotunde AA (2014) On the joint optimization of well placement and control. In: SPE 172206

Awotunde A, Naranjo C (2014) Well placement optimization constrained to minimum well spacing. In: SPE 169272

Bagheri M, Masihi M (2016) Investigating the effect of heterogeneity on infill wells. J Pet Explor Prod Technol 6(3):451-463

Bangerth W, Klie H, Wheeler M, Stoffa P, Sen M (2006) On optimization algorithms for the reservoir oil well placement problem. Comput Geosci 10(3):303-319

Bouzarkouna Z, Ding D, Auger A (2012) Well placement optimization with the covariance matrix adaptation evolution strategy and meta-models. Comput Geosci 16:75-92

Cao X, Guan Z, Shi Y, Xue L, Zhang X (2016) Drilling sequence optimization model and its solution method for offshore cluster wells. J China Univ Pet 40(3):96-101

Carosio G, Humphries T, Haynes R, Farquharson C (2015) A closer look at differential evolution for the optimal well placement problem. In: Proceedings of the 2015 annual conference on genetic and evolutionary computation, pp 1191-1198

Chen H, Feng Q, Zhang X, Wang S, Zhou W, Geng Y (2017a) Well placement optimization using an analytical formula-based objective function and cat swarm optimization algorithm. J Pet Sci Eng 157:1067-1083

Chen H, Feng Q, Zhang X, Zhou W, Geng Y (2017b) A prediction formula for ratio of injection-production control area in triangle well pattern. J Pet Explor Prod Technol. https://doi.org/10.1007/ s13202-017-0330-6

Clarke-Sather A, Qu J, Wang Q, Zeng J, Li Y (2011) Carbon inequality at the sub-national scale: a case study of provinciallevel inequality in $\mathrm{CO}_{2}$ emissions in China 1997-2007. Energy Policy 39(9):5420-5428

Dossary M, Nasrabadi H (2016) Well placement optimization using imperialist competitive algorithm. J Pet Sci Eng 147:237-248

Feng Q, Wang X, Wang D, Huang Y (2016) Theoretical analysis on the performance of equilibrium displacement in water flooding reservoir. Pet Geo Recovery Effic 23(30):83-88

GeoQuest S (2010) ECLIPSE reference manual 2010.1. Schlumberger Inf. Solutions, Houston, Tex

Huang V, Qin A, Suganthan P (2006) Self-adaptive differential evolution algorithm for constrained real-parameter optimization. IEEE Trans Evolut Comput 1:17-24

Humphries T, Haynes R (2015) Joint optimization of well placement and control for nonconventional well types. J Pet Sci Eng $126: 242-253$ 
Isebor O, Durlofsky L, Ciaurri D (2014) A derivative-free methodology with local and global search for the constrained joint optimization of well locations and controls. Comput Geosci 8(3-4):463-482

Jesmani M, Bellout M, Hanea R, Foss B (2016) Well placement optimization subject to realistic field development constraints. Comput Geosci 20(6):1185-1209

Khademi G, Karimaghaee P (2016) Hybrid FDG optimization method and kriging interpolator to optimize well locations. J Pet Explor Prod Technol 6(2):191-200

Li Y, Wang D, Li C (2006) Vectorial well arrangement in anisotropic reservoir. Pet Explor Dev 33(2):225-227

Marir B, Mandel C, Jefri A, Torres J, Ferdian S (2015) Optimization of well trajectories and wellhead towers' slots allocation through a collaborative well-planning approach. In: SPE 172565

MATLAB R (2013) Version 8.1. 0.604 (R2013a). The MathWorks Inc, Natick

Nwankwor E, Nagar A, Reid D (2013) Hybrid differential evolution and particle swarm optimization for optimal well placement. Comput Geosci 17:249-268

Ogbeiwi P, Aladeitan Y, Udebhulu D (2017) An approach to waterflood optimization: case study of the reservoir X. J Pet Explor Prod Technol. https://doi.org/10.1007/s13202-017-03685

Padilla E, Serrano A (2006) Inequality in $\mathrm{CO}_{2}$ emissions across countries and its relationship with income inequality: a distributive approach. Energy Policy 34(14):1762-1772
Siavashi M, Tehrani M, Nakhaee A (2016) Efficient particle swarm optimization of well placement to enhance oil recovery using a novel streamline-based objective function. J Energy Resour 138(5):77-78

Taware S, Park H, Datta-Gupta A, Bhattacharya S, Tomar A, Kumar M, Rao H (2012) Well placement optimization in a mature carbonate waterflood using streamline-based quality maps. In: SPE 155055

Wang X, Haynes R, Feng Q (2016) A multilevel coordinate search algorithm for well placement, control and joint optimization. Comput Chem Eng 95:75-96

Yeten B, Durlofsky L, Aziz K (2002) Optimization of nonconventional well type, location and trajectory. In: SPE 77565

Zhang Y, Lu R, Forouzanfar F, Reynolds A (2017) Well Placement and control optimization for WAG/SAG processes using ensemble-based method. Comput Chem Eng 101:193-209

Zhou Z, Wang J, Zhou J (2017) Research on the overall optimization method of well pattern in water drive reservoirs. J Pet Explor Prod Technol 7(2):465-470

\section{Publisher's Note}

Springer Nature remains neutral with regard to jurisdictional claims in published maps and institutional affiliations. 